\title{
A case study from the Sunnybrook and Women's College Health Sciences Centre: Do clinical trials of neoadjuvant chemotherapy for locally advanced breast cancer ( $L A B C)$ overestimate the actual response rates observed in clinical practice?
}

\author{
M. Clemons, M. Trudeau \\ Division of Medical Oncology/Hematology, Sunnybrook and Women's College Health Sciences Centre, \\ Toronto, Ontario, Canada.
}

\section{Case report}

A 48-year-old premenopausal woman was referred to our locally advanced breast cancer (LABC) programme by her surgeon. She presented with a 4month history of a slowly growing mass in the left breast. Assessment by the surgeon was suspicious for malignancy and a core biopsy confirmed the presence of an oesterogen receptor (ER) and progesterone receptor (PR) negative, HER2/neu negative breast cancer. Staging chest X-ray, liver ultrasound, and bone scan were all normal. On clinical examination the left breast was replaced with tumour. It was fixed to skin with peau d'orange but was not fixed to the chest wall. The tumour within the breast measured $10 \times 8 \mathrm{~cm}$. The patient also had mobile but matted axillary lymph nodes measuring $5 \times 1 \mathrm{~cm}$.

She therefore had clinical T4b, N2a (stage IIIB) disease. She was treated on a standard protocol of 3 weekly AC for 4 cycles followed by 3 weekly taxotere for 4 cycles. This was to be followed by surgery and full field radiotherapy to both the chest wall and local lymph node sites. After completion of chemotherapy, examination of her breasts revealed a $5 \mathrm{~cm}$ diameter

\footnotetext{
Correspondence to: Dr Maureen Trudeau, BSc, MA, MD, FRCPC, Head, Division of Medical Oncology/Hematology, Sunnybrook and Women's College Health Sciences Centre, Associate Professor, University of Toronto; Sunnybrook and Women's College Health Sciences Centre, Division of Medical Oncology/Hematology, 2075 Bayview Avenue, Toronto, Ontario M4N 3M5, Canada. E-mail: Maureen.Trudeau@sw.ca; Tel: +1 416 480 5145; Fax: +14164806002

Publication date 29/04/05 BCO/81/2002/CS
}

soft thickening in her left breast and no palpable axillary lymphadenopathy. Pathology assessment after mastectomy showed a residual $3 \mathrm{~cm}$ tumour, Bloom and Richardson grade 2/3, in the breast. Single tumour cells and an occasional group of tumour cells were present within the epidermis and no lymph nodes were involved. All resection margins were clear.

The patient wanted to know what additional systemic therapy was needed in view of her residual disease at the time of mastectomy.

\section{Comment}

Neoadjuvant chemotherapy has been widely tested in recent years. The goals of neoadjuvant therapy however vary between patients. The greatest potential benefit is the opportunity to offer some patients breast conserving surgery. This was unlikely to be an option in this patient due to the characteristics of the tumour at presentation.

While survival has been shown to be identical for operable breast cancers with pre-operative or standard adjuvant therapy [1] another potential benefit is the demonstration of in vivo tumour sensitivity to chemotherapy. Evidence shows that both clinical (cCR) and pathologic (pCR) complete response predicts for locoregional recurrence, disease free and overall survival. Phase II and III trials with different neoadjuvant regimens report cCR rates of $60-80 \%$ with corresponding pCR rates of $10-39 \%[1,2]$. We recently used the prospective data base from our own LABC programme to compare how clinical and 
pathologic response rates in a non-trial setting compared with the response rates reported in the literature for women with $L A B C$.

A cohort of 34 patients with $L A B C$ were treated between September 2003 and April 2004. Breast cancer patients with clinical stage IIIA (T3N1; T0-2N2), IIIB (T4NO-2; any T0-4N3) and primary tumours greater than $5 \mathrm{~cm}$ were defined as having $\mathrm{LABC}$ and were eligible for the study. Two patients were excluded as they did not complete neoadjuvant chemotherapy. The median patient age was 49 (range 29-71). Clinical staging at diagnosis showed 15\% IIB, 47\% IIIA, 32\% IIIB and 6\% IIIC. Eighty-five per cent were clinical stage III at presentation and of these, four (12\%) had T4d inflammatory breast cancers. Eighty-eight per cent had clinical axillary node involvement. Fifty-five per cent of tumours were hormone receptor positive and $38 \%$ were positive for HER2/neu overexpression. Twenty-four patients (71\%) received an anthracycline-based regimen. Following neoadjuvant chemotherapy, $27 \%$ of patients had a cCR and $35 \%$ had a partial response (cPR), for a total clinical response rate of $62 \%$. Pathologic complete response was seen in 2 patients (6\%).

There is clearly a major difference in cCR and pCR between clinical trials and our own dataset. The cCR and $\mathrm{pCR}$ response rates in this study were $27 \%$ and $6 \%$, respectively. This is much lower than reported in clinical trials and likely reflects the advanced nature of patients selected for neoadjuvant therapy in actual practice. It also leads to the important question as to whether or not additional chemotherapy after surgery could lead to an improvement in patient outcome when residual disease is found despite neoadjuvant therapy. Our own group policy is not to give further post-operative chemotherapy in these patients provided they have received adequate neoadjuvant therapy.

This important question should be asked in the clinical trial setting.

\section{References}

1. Fisher B, Bryant J, Wolmark N, et al. Effect of preoperative chemotherapy on the outcome of women with operable breast cancer. J Clin Oncol 1998; 16: 2672-2685.

2. Bear HD, Anderson S, Brown A, et al. The effect on tumor response of adding sequential preoperative docetaxel to preoperative doxorubicin and cyclophosphamide: preliminary results from National Surgical Adjuvant Breast and Bowel Project Protocol B-27. J Clin Oncol 2003; 21: 4165-4174. 